\title{
Workshop proposal: introduction to automatic audio classification
}

\author{
Tiago Fernandes Tavares $^{1 *}$ \\ ${ }^{1}$ School of Electric and Computer Engineering - University of Campinas \\ Av. Albert Einstein, 400 - 13083-852 - Campinas, SP \\ tavares@dca.fee.unicamp.br
}

\begin{abstract}
This hands-on workshop comprises essential techniques for digital signal processing and machine learning. Participants will use the Python libraries librosa and scikit-learn as support to build an automatic audio classification system. The workshop will use explorations in toy problems to approach theoretical aspects. Later, it will discuss practical issues for building a scientific applications in the field.
\end{abstract}

\section{Program}

1. Introduction to Python3 and visualization of audio signals in the time domain,

2. Frequency-domain visualizations and spectrograms,

3. Calculating and visualizing spectral shape descriptors,

4. Mapping audio signals to vectors,

5. Machine learning algorithms and pipelines (normalization, KNN, SVM),

6. Evaluating audio classification systems,

7. Discussion on the problem of audio tagging.

\section{Target audience}

- Up to 20 participants.

- Previous experience with Python is desirable, but knowledge on any computer language is quickly transferable to Python.

- Participants with no experience in programming are welcome, but their progress will likely be a little slower.

\section{Required Infra-Structure}

This is a hands-on workshop based on computer programming. As such, it requires one computer per participant. The computers can run on any operating system, and must have Internet access. The workshop will use online-hosted resources, hence no local configuration is required.

Ideally, a dedicated computer laboratory (with desktop computers) will be used. Optionally, it is possible to ask participants to bring their own laptops, as long as a reliable wi-fi connection is provided.

\footnotetext{
*Supported by CNPq.
} 